\title{
A review of the Centre for Evidence-based Dentistry website
}

\author{
Shuva Saha \\ Specialist Registrar in Restorative Dentistry, Birmingham Dental Hospital, Birmingham, UK
}

Evidence-Based Dentistry (2005) 6, 80-82. doi:10.1038/sj.ebd.6400352

\section{Introduction}

The Centre for Evidence-based Dentistry (CEBD) is a UK-based organisation initially established in 1995 in Oxford. Its website was developed to promote evidence-based dentistry (EBD) and to provide its users who have an interest in the subject with a comprehensive range of high-quality resources to enable them to understand and practice evidence-based dentistry. Its target audience is wide ranging and it is a central resource point for both dental professionals and the public.

Its website has been running for 10 years and has recently undergone major restructuring and now provides clearer navigation with important new sections on How to Practice Evidence-based Dentistry and Conducting Research. It can be viewed at www.cebd.org. The aims of this website are to develop and disseminate resources that are suitable for integrating evidence into dental clinical practice. Although the ideas behind evidencebased practice have been with us for a long time, there has been a recent interest in applying evidence-based principles into practice and this website has been set up to promote this.

\section{Layout}

The overall presentation of this site is visually appealing and professional looking. The screen displays are uncluttered and concise. One is able to gain immediately a clear indication of the purpose of this website and the service that it aims to provide. Although the website is extensive and delivers information on a variety of topics, it is well organised into the relevant subject areas and remains user friendly. The website is easy to navigate and at the same time allows users to maintain their bearings during their virtual visit. Link buttons to the core pages are arranged in a logical order at the top of each page.

\section{Content}

The Home page (Figure 1) provides a direct link to the journal of EBD which aims to deliver the best available evidence on the latest developments in oral health. For nonsubscribers to the journal, abstracts are available from the featured articles. Clicking on What is EBD leads to

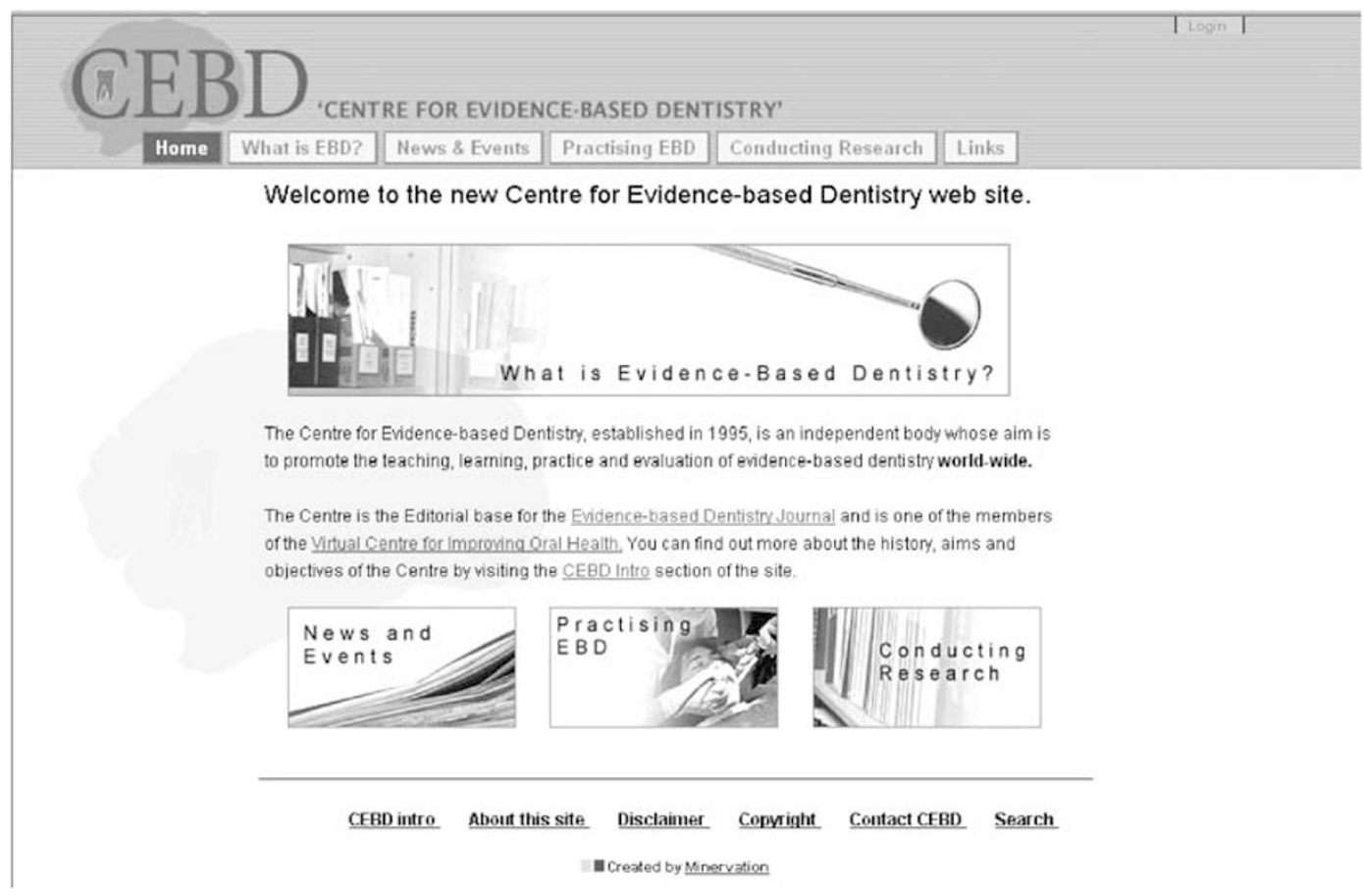

Figure 1. Centre for Evidence-based Dentistry — Home page. 


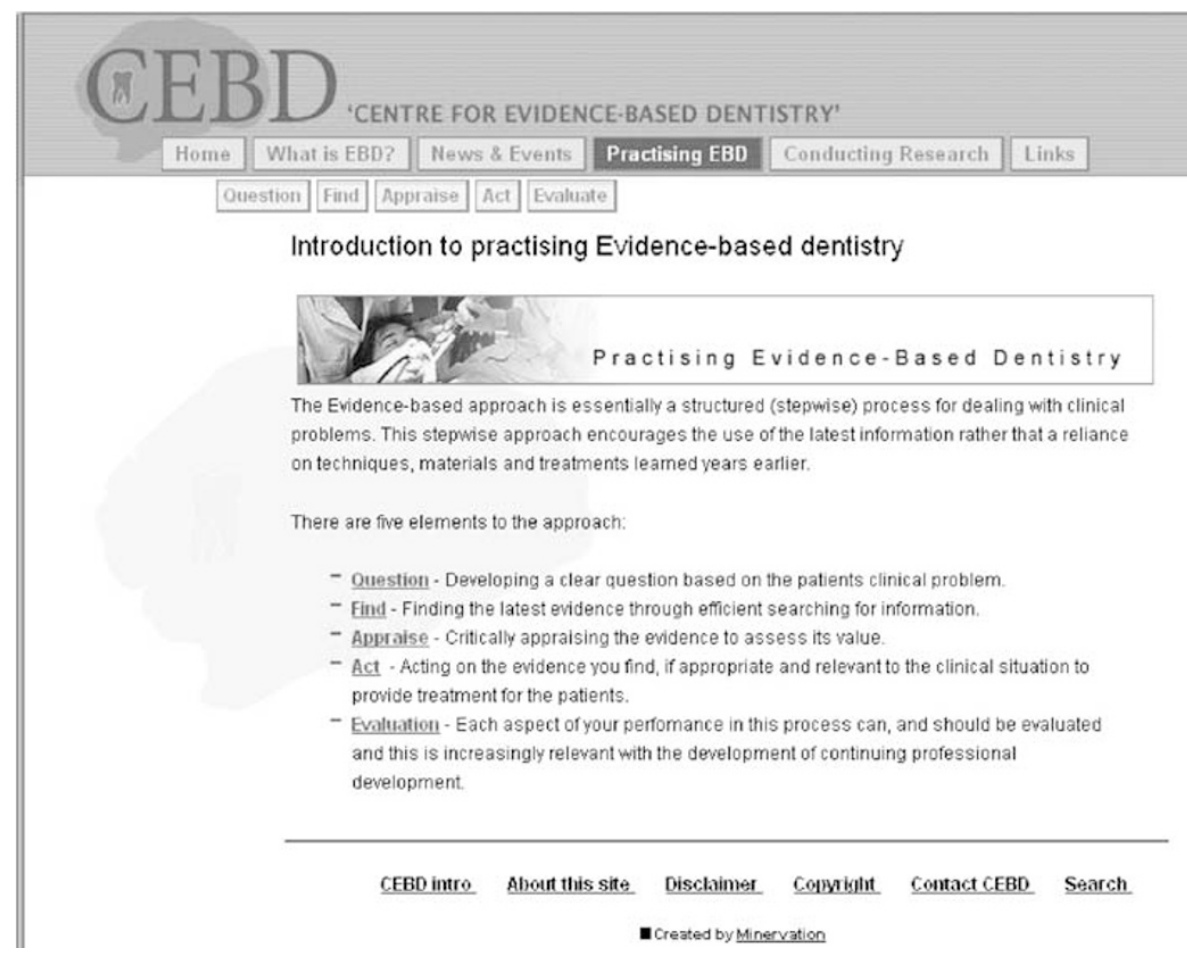

Figure 2. Practising Evidence-based dentistry page.

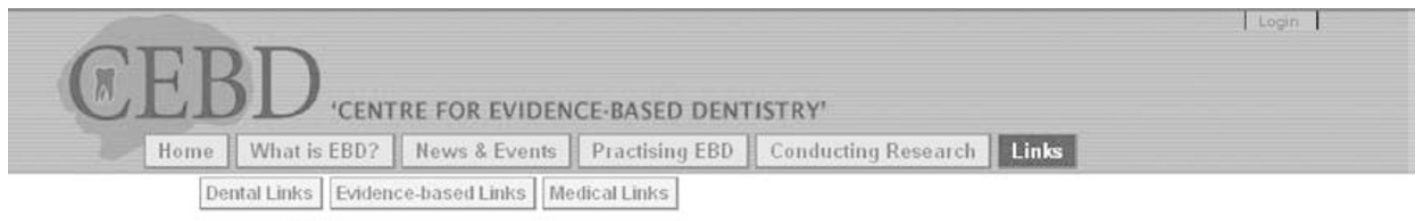

Links
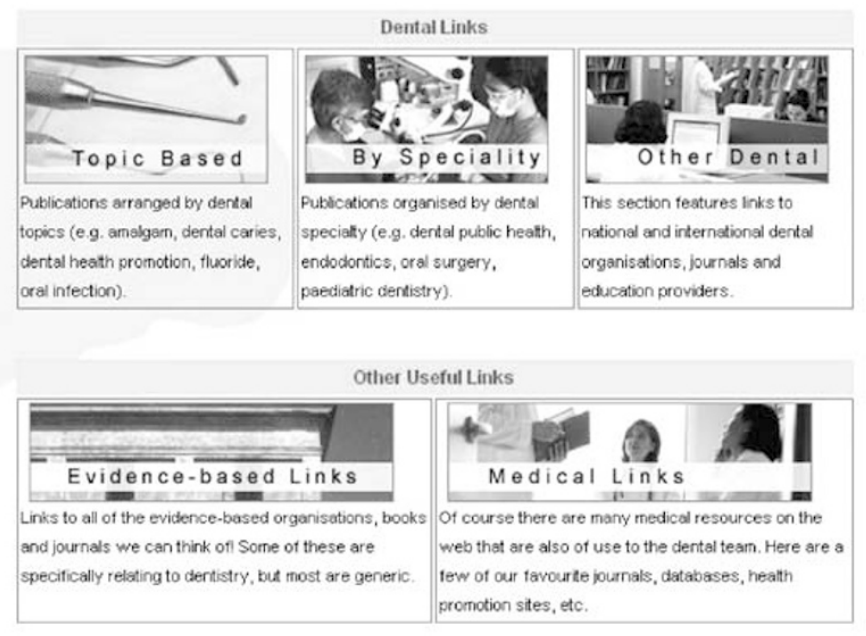

CEBD intro About this site Disclaimer Coprriglth Contact CEBD Search

$$
\text { in Created by Minervation }
$$

Figure 3. Centre for Evidence-based Dentistry — Links page. 
clear definitions of the topic and provides a list of the relevant EBD references. There are direct links to full text or pdf versions of these references. The News and Events page provides information on the relevant latest happenings and in the world of EBD and keeps the user informed and updated. In the events list, there are details of significant educational courses, conferences and seminars taking place nationally and internationally. There is also the opportunity to sign up for a newsletter distributed via e-mail; however, distribution of the newsletter is not planned until the end of the year.

The Practising Evidence-Based Dentistry page (Figure 2) contains a comprehensive, structured process for dealing with clinical problems. Each element to the approach is clearly dealt with an extensive list of links. For anybody wishing to conduct their own research, the Conducting Research section provides a very brief introduction to some of the more common research designs with links to various useful resources. The introduction to this section has a direct link to the Cochrane Oral Health Group, with subdivisions into the various clinical study types. These are supported with brief descriptions and links to an extensive list of resources.

The Links page (Figure 3) has been subdivided into five different areas. Within these subdivisions all aspects of evidence-based dentistry are covered including many medical resources. In reality, it seems impossible to think of any relevant site that does not have an active link from the CEBD website.

\section{Conclusion}

Information overload has become one of the most significant problems that internet users face. The content can be highly variable, clinical information is not separated from nonclinical information and despite several high-quality clinical websites there is still a large amount of information of little use on the web. With such a vast quantity of information at hand, finding information or the answer to a specific question can be tricky.

The information provided on the Centre for Evidence-based Dentistry website is of high quality and this site will help users find the best information quickly, assess its quality, decide whether it is relevant and help use research evidence in making all important everyday clinical decisions. It is a well-developed, highly reliable online resource that provides excellent evidence-based information. It boasts a wealth of authoritative information on almost anything connected with evidence-based dentistry. The Centre for Evidence-based Dentistry website is an essential tool for anybody wanting to use an evidence based approach to deal with clinical issues. 